\title{
The Impact of The Implementation of Gradual Run Warming Up toward The Development of The Cardio Endurance Ability
}

\author{
Fitri Rosdiana ${ }^{1}$, Dikdik Zafar Sidik $^{2}$, Yudi Nurcahya ${ }^{3}$ \\ \{fitrirosdiana@upi.edu ${ }^{1}$,dikdikzafarsidik@upi.edu ${ }^{2}$, udinurcahya@upi.edu ${ }^{3}$ \} \\ Universitas Pendidikan Indonesia, Bandung, Indonesia ${ }^{1,2,3}$
}

\begin{abstract}
Warming up is a preliminary training or exercise to prepare the body for physical activities by gradually increasing the intensity (increasing the pulse). The goal of this research is to determine the impact of the implementation of gradual run warming up toward the development of the cardio endurance ability. This research is an experimental research with One-Group Pretest-Posttest design. The sample was 40 Sports college students. They were selected by purposive sampling. The research instrument to determine the cardio endurance ability was Balke Test and the data analysis technique was SPSS version 21 software. SPSS is a computer software whose function is to calculate statistical data. The results showed that the implementation of gradual run warming up could have a significant influence on the development of the cardio endurance ability.
\end{abstract}

Keywords: warming up, continuous run, cardio endurance, balke test

\section{Introduction}

In general, sports are very beneficial for health because they can improve the quality of one's body. Any type of training cannot be separated from the needs for good physical fitness. Physical fitness is the ability to perform daily activities with enthusiasm [1] without experiencing excessive fatigue [2] and allows the athletes to recover before doing further activities [3]. Physical fitness, physiologically, consists of anaerobic and aerobic abilities. Aerobic ability is often also called as general endurance [3]. Cardiorespiratory endurance is the ability of the circulatory system to provide and store (supply) energy during the activity so that it does not encounter fatigue [4]. Cardiorespiratory endurance is a place in which adenosine triphosphate (ATP) is re-synthesized to produce energy. This activity usually lasts longer than five minutes [5]. In sports, endurance has a very important role in every branch of sport. Before doing physical fitness activities, endurance needs to be warmed up first.

Warming up is a preliminary training prepared to improve further physical training [6]. Warming up must be specific to the physical activity that will be carried out $[7,8]$. Warming up is useful for increasing body temperature, expediting blood flow, warming muscle temperature, improving muscle contraction, and increasing coordination [9]. Warming up will reduce and prevent the possibility of injury to the muscles [10]. 
According to [11] warming up techniques can be categorized into two: passive and active. There are three types of warming up methods, namely passive, general, and specific [6]. Passive warming up is a warming up that is done to increase body temperature (muscle temperature) by external manners such as taking saunas, bathing using hot water, and having massage [11, 12]. Active warming up involves physical activities that cause greater cardiovascular changes such as jogging, running, cycling, skipping, gymnastics, and others [13]. In accordance to [14] that general warming up such as jogging followed by stretching of certain muscles is included in active warming up. Specific warming up includes special movements that are part of physical activity or mimic the movements that will be carried out in the core training [8].

Traditionally, stretching has been a part of the warming up process [15]. There are three methods of stretching according to [16], namely static, dynamic, and PNF (Propeoceptive neuromuscular Facilitation). Firstly, static stretching is done slowly to achieve muscle elongation [17]. The increase of the length of the hamstring muscles when using static stretching can be maintained for up to 24 hours [18]. Static stretching involves passive stretching which is held for a long time [19]. Secondly, dynamic stretching techniques are performed by bouncing and swinging rhythmically [16]. Each stretching is maintained for 20 seconds. Furthermore, the third stretching technique, PNF, is commonly used to increase Range of Motion (ROM) and relax muscles [20]. PNF is the most difficult stretching which requires guidance since this stretching combines several techniques [16].

According to [21], stretching method consists of three. The first one is static stretching. This stretching is divided into two: active static and passive static. Active static is done by yourself statically (holding still), followed by passive static with the help of others. The second one is dynamic stretching which is carried out by moving the body parts in a rhythmic (dynamic). Meanwhile the last stretching is PNF. Some ways to conduct PNF are contractionrelaxation technique, relaxation-contraction technique, contraction-relaxation-contraction techniques, contraction-relaxation-antagonist contractions, and hold-relax-swing technique.

The purpose of warming up is to raise body temperature and performance which consists of aerobic activities with submaximal intensity, like running and cycling [22, 23, 24]. Running is an accelerated frequency of steps so that when we are running, there is a tendency for the body to float. It means that our feet do not touch the ground or at least one foot still touches the ground [25]. The warming up implemented in this research was gradual run warming up which was started at the beginning of the meeting as much as 4 rounds with a distance of 400 $m$ track and the warming up program was increasingly improved with progress (4-4-5-5-6-67-7- 8-8-9-9-10-10-11-11). A lot of relevant research stated that a performance could be improved by warming up [26]. The attempt to warm up before doing the training actually has a beneficial effect on the endurance with an indicator that sweat is coming out [27]. Athletic performance according to coaches and athletes could be improved by warming up. In addition, warming up could reduce the likelihood of injury [28]. A performance could be improved by warming up regardless of the intensity of the warming up itself [29]. This research shows that completing warming up before doing the next training has been shown to improve the subsequent performance. Improvements have been seen in aerobic and anaerobic sports; one of them is running [30]. 


\section{Methods}

\subsection{Subjects}

The population and sample in this research were 40 Sports college students batch 2018 . They were selected by purposive sampling technique. After receiving detailed explanation of the objectives, potential benefits, and risks associated with participating in this research, each student gives his written consent.

\subsection{Protocol}

In its implementation, researchers conducted the initial test and measurement as initial data and then gave them gradual run warming up treatment. After finishing the treatment, the final stage of the test and measurement was carried out to determine the results of the warming up program that had been given. The step taken for data collection was preparing the test instruments. The data were in the form of quantitative. The data collection schedule consisted of two stages. Those stages were a preliminary test to determine the initial conditions of the subject and a final test to see the progress of the results of the training treatment.

The method was an experimental method with one group pretest-posttest design [31]. The research instrument was gradual run warming up training program as the process in collecting the data and test items to determine the ability of cardio endurance; that was measured through the Balke Test [32].

\section{$3 \quad$ Results and discussions}

The data gathered from the sample in conducting pretest and posttest were analyzed by SPSS version 21 software. The analysis technique was paired sample t-test. This test was used to test whether there were differences or influences. The first step to see the impact of the implementation of gradual run warming up toward the development of the cardio endurance ability was to do a normality test though the Kolmogorov-Smirnov One-Sample Test. Because the distribution of the subjects was normal, it was then followed by a descriptive data test through Paired Samples Statistics and a correlation test though Paired Samples Correlations.

Table 1. Gradual run warming up

\begin{tabular}{cccccc}
\hline \multirow{2}{*}{ Component } & \multicolumn{4}{c}{ Gradual Run Warming Up } \\
\cline { 2 - 4 } & \multicolumn{3}{c}{ Mean } & \multirow{2}{*}{ Correlation } & \multirow{2}{*}{ Sign } \\
\cline { 2 - 5 } & Before & After & & .816 & .000 \\
\hline Cardio Endurance Ability & 13.62 & 12.82 & & .62 \\
\hline
\end{tabular}

The average value of cardio endurance ability pretest and posttest is $13.62>12.82$., which means that there is an average difference between pretest and posttest trainings and there is also the correlation coefficient value of cardio endurance which is 0.816 with a significance 
value of 0.000 . Because the Sig value <probability 0.05 , it can be said that there is a relationship between pretest and the posttest variables. To prove whether the difference is really significant, then we need to interpret the results of the paired sample $T$ test contained in Table II.

Table 2. Results of the paired sample T test

\begin{tabular}{rccc}
\hline \multirow{2}{*}{ Component } & \multicolumn{3}{c}{ Gradual Run Warming Up } \\
\cline { 2 - 4 } & Result & Sign. & Significance Test \\
\hline Cardio Endurance Ability & Increased & .000 & Significant \\
\hline
\end{tabular}

Based on the test table above, the Sig. (2-tailed) of the cardio endurance is $0,000<0.05$, then $\mathrm{HO}$ is rejected and $\mathrm{Ha}$ is accepted. Therefore, it can be concluded that there is an average difference between pretest and posttest, which means that there is an effect of the gradual run warming up toward the development of the cardio endurance.

The development can be seen in the table below.

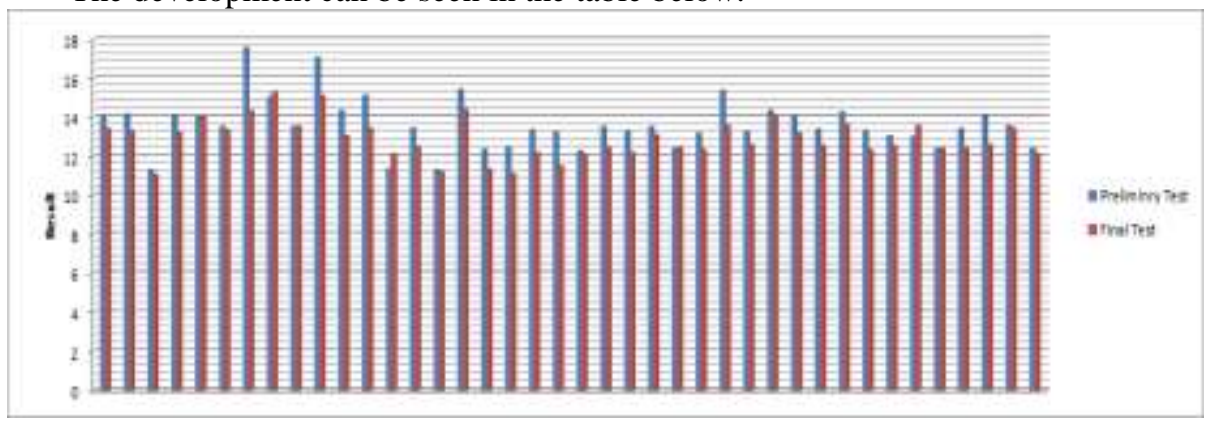

Fig. 1. Percentage of initial test and final test of the cardio endurance ability affected by the gradual run warming up

From the results above, it was found that the process of the implementation of gradual run warming up affects the development of the cardio endurance ability. The results of this research are supported by $[22,23,24]$ that the warming from aerobic activities with submaximal intensity, such as running and cycling, could raise body temperature and performance. The warming up must be in line with the principle of specifications if the coaches, trainers, or even researchers want increase in the obtained results to be more effective. This is because specific warming up will affect the next physical activity that will be carried out. [7, 8] stated that the performed warming up must be specific to the physical activity to be carried out.

The results of this research indicate that the effect is not too large. This can be seen from a few samples who only experienced a slight increase because the sample has better cardio endurance quality hence their maximum ability is almost achieved. Changes in the initial test and the final test on the gradual run warming up toward the cardio endurance are in accordance with physiological principles because the increasing number of repetitions, the longer duration of doing, and the increasing distance traveled will give a better adaptation effect. In addition, the discipline factor of the sample in undergoing the provided training 
program will also affect the results. [33] said that athletes who carry out training programs in a disciplined manner with intense training could get the benefit from the exercise.

\section{Conclusion}

Researchers have reported various results regarding the effect of warming up. In this research, it can be said that the implementation of gradual run warming up gives a significant influence toward the development of the cardio endurance ability. For further research, it is recommended that the topic of his research have to be adapted to the needs of further training. In the other words, the warming up must be specific in accordance with the sport so that the benefits of the warming up can be well-achieved because the warm up will affect the next physical activity that will be carried out. In addition, for further research, researchers suggest that it should not only be based on the distance traveled but time and intensity also need to be considered.

\section{References}

[1] Hedrick, A. Physiological responses to warm-up. J Strength Cond Res, 1992. 14: 25-27

[2] Shellock, F. G, Prentice, W. E.Warming-up and stretching for Improved physical performance and prevention of sports-related injuries. 1985. pp 267-278

[3] Faigenbaum $\mathrm{AD}$, Bellucci M, Bernieri A, et al. Acute effects of different warm-up protocols on fitness performance in children. J Strength Cond Res; 2005.19 (2): 376-381

[4] Smith, CA. The warm-up procedure: To stretch or not to stretch. A brief review. J Orthop Sports Phys Ther 2004. 19: 12-17.

[5] Safran M.R, et al. The role of warmup in muscular injury prevention.(january), 1988. pp: 123-129

[6] Bishop, D. Performance changes following active warm up and how to structure the warm up. 2003. 33 (7): 483-498

[7] Safran MR, Seaber AV, Garrett Jr WE. Warm-up and muscular injury prevention: an update. 1989. 8 (4): $239-250$

[8] Wathen, D. Flexibility: Its place in warm up activities. NSCA Journal. 1987. 6:26-27

[9] Young, W. B and Behm, D. Effect of running static streching and practice jumps on explosive force production and jumping performance. 2003. (March)

[10] Carolina et al. Effect of Warm-Up on the Standing Broad Jup, 2003. 17 94), pp 710-714

[11] Yokoi, H and Campisi, P. Effect of streching on Prevention of Injuries for Military Recruits. 2018. 168 (August 2002), pp 442-446

[12] Woods, K, Bishop, P, and Jones, E. Warm Up and Streching in thr Prevention of Muscular Injury, 2007. 37(12)

[13] Volker, C et al. The Effect of Static Stretch and Warm-up Exercise on Hamstring Length Over the Course of 24 Hours. J Orthop Sports Phys Ther 2003;33:727-733.

[14] Ma, J. E. B and Beaulieu, J. E. Streching Program, 2016. 3847 (December). doi: 10.1080/00913847.1981.11711207.

[15] Osternig LR, Robertson R, Troxel R, Hansen P. Muscle activation during proprioceptive neuromuscular facilitation (PNF) stretching techniques. American Journal of Physical Medicine. 1987. Oct;66(5):298-307

[16] Sidik, D. Z et al. Pelatihan Kondisi Fisik. Bandung : PT Remaja Rosdakarya 2019. ISBN 978-602446-343-4 
[17] Young W, Behm D. Should static stretching be used during a warm-up for strength and power activities? Strength Cond 2002. J24:33-37

[18] Young, W. B. The use of Statistic Stretching in warm up for training and Competition, 2007, 212216)

[19] Behm, D and Chaouachi, A. A review of the acute effect of static and dynaic streching on performance, 2011. doi : 10.1007/s00421-011-1879-2.

[20] Djumidar,A. W. M. Belajar Berlatih Gerak-Gerak Dasar Atletik dalam Bermain. Jakarta: PT Raja Grafindo, 2004

[21] Fradkin, AJ, Zazryn, TR, and Smoliga, JM. Effects of warming-upon physical performance: a systematic review with metaanalysis.J Strength Cond Res 2010, 24(1): 140-148.

[22] Chwalbii, J. Effect of Active Warming-up on Thermoregulatory , Circulatory , and Metabolic Responses to Incremental Exercise in Endurance-Trained Athletes *, 1898, 0-4.

[23] Grodjinovsky and Magel. Research Quartely. American Association for Health, Physical education and Recreation effect of warm-up running performance. (april 2015) pp 37-41.

[24] Hajoglou, et al. Efect of warm-up on Cycle Time Trial performance. 2005. Doi:10.1249/01.mss.0000177589.02381.0a

[25] McBride, JM, Nimphius, S, and Erickson, TM. The acute effects of heavy-load squats and loaded countermovement jumps on sprint performance. J Strength Cond Res 2005, 19: 893-897

[26] Fraenkel J. R. dan Wellen, N. E. How to Design and Evaluate Research in Education (Second edition). United States of America: Mc-Graw Hill,inc, 1993.

[27] Brian Mckenzine. 101 Performance Evaluation Test. 2005. ISBN: 1-905096-18-6

[28] Iaia, F. M. and Bangsbo, J. Speed endurance training is a powerful stimulus for physiological adaptations and performance improvements of athletes. Scandinavian Journal of Medicine and Science in Sports, 20(SUPPL. 2), 2010, pp. 11-23. doi: 10.1111/j.1600-0838.2010.01193.x. 